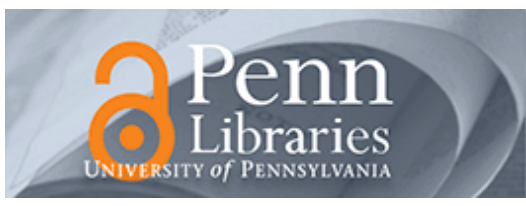

University of Pennsylvania
ScholarlyCommons

Departmental Papers (ASC)

Annenberg School for Communication

2005

\title{
A Call to Action on Adolescent Mental Health
}

\author{
Kathleen Hall Jamieson \\ University of Pennsylvania, kjamieson@asc.upenn.edu \\ Daniel Romer \\ University of Pennsylvania, dromer@asc.upenn.edu
}

Follow this and additional works at: https://repository.upenn.edu/asc_papers

Part of the Health Communication Commons

\section{Recommended Citation (OVERRIDE)}

Jamieson, K. H., and Romer, D. (2005). "A Call to Action on Adolescent Mental Health" in D. L. Evans, et. al., (eds.), Treating and Preventing Adolescent Mental Health Disorders: What We Know and What We Don't Know (pp. 617-623). New York, Oxford University Press.

This paper is posted at ScholarlyCommons. https://repository.upenn.edu/asc_papers/710

For more information, please contact repository@pobox.upenn.edu. 


\section{A Call to Action on Adolescent Mental Health}

\section{Disciplines}

Communication | Health Communication | Social and Behavioral Sciences 


\section{A Call to Action on Adolescent Mental Health}

Kathleen Hall Jamieson and Daniel Romer 
In this final chapter, we summarize important policy implications of the commission reports and highlight steps they suggest to advance the healthy development of America's youth. The reports provide a hopeful assessment of our ability to treat the most prevalent adolescent disorders. At the same time, enormous hurdles remain in our ability to deliver these treatments, and our knowledge base of effective treatments still has important gaps. These considerations suggest that we face formidable challenges if we wish to ensure the healthy development of our youth. Nevertheless, our ever-growing understanding of environments that encourage healthy development bodes well for our future ability to both treat and prevent adolescent mental disorder.

\section{TREATMENT WORKS}

The good news in the commission reports is that the most common disorders (anxiety and depression) have effective treatments that can help more than $70 \%$ of those who are afflicted. Those who do not respond to particular treatments can be given alternative therapies that can raise the success rate even higher. Although combination treatments involving both drugs and psychotherapy are often most effective, it is also the case that psychotherapy, in particular cognitive-behavior (CBT) or interpersonal (IPT) approaches, can reduce symptoms and lead to improvement without the use of medication.

There is also progress in the treatment of the less prevalent conditions. Treatments for bipolar disorder have a high success rate, and therapeutic interventions for anorexia nervosa can lead to recovery from this illness. Early intervention can also benefit those with schizophrenia, reducing the severity of the illness and leading to better adaptation to the disorder. Since many with severe mental disorders are at risk for suicide, these interventions can be not only life altering but also life saving.

As discussed below, the findings also have important implications for the reduction of stigma associated with mental illness. Public awareness of the effectiveness of treatment for mental disorders should increase the willingness of parents and youth to seek treatment before illness pro- gresses. Stigma reduction throughout society should also increase the likelihood that those who have been successfully treated will lead productive and satisfying lives.

\section{RECENT DEVELOPMENTS IN THE USE OF MEDICATION TO TREAT ADOLESCENT DEPRESSION}

As this book goes to press, there is vigorous discussion about the safety and efficacy of antidepressants in particular, selective serotonin reuptake inhibitors (SSRIs), for treatment of adolescent depression. Many clinical trials supported by the pharmaceutical industry suggest the potential for adverse events in the use of SSRIs, including increased suicidal ideation and suicide attempts (Harris, 2004a, b). Unfortunately, these trials were often kept from public view. This has led to increased pressure to make all clinical trials involving drugs available for public inspection. In addition, an FDA panel has determined that these trials support the conclusion that SSRIs may carry an increased risk for suicidal ideation or behavior for a small proportion of users (perhaps 2 or 3\%) and as a result labels warning of these effects will now be placed on all antidepressants (FDA, 2004).

A recent trial with adolescents suffering from major depression (Glass, 2004; March et al., 2004) indicated that treatment with a particular SSRI (Prozac) was less likely to produce adverse events when combined with CBT. Based on this evidence as well as considerable research suggesting the effectiveness of CBT as well as IPT with adolescents (reviewed by the depressionbipolar commission), it may be that combined treatment is the best approach for adolescents who present with depression and suicidal thoughts or behaviors. Since many physicians and mental health providers may not be trained to deliver CBT or IPT, there is a clear need to increase the number of practitioners who can provide these alternatives. Use of CBT or IPT may be the preferred alternative for less severe cases, since it has proven efficacy and does not run the risk of increased suicidal behavior.

It is encouraging to note the appearance of several initiatives designed to increase public ac- 
cess to the results of clinical trials evaluating the effects of therapeutic interventions. Eleven major medical journals have established a policy requiring the registration of such trials at inception before findings can be considered for publication (DeAngelis, Drazen, Frizelle, Haug, Hoey, Horton, et al., 2004). The American Medical Association has endorsed the concept of a federally mandated registry of clinical trials (Council of Scientific Affairs, 2004). Legislation mandating registration has been introduced in both the House and Senate (Fair Access to Clinical Trials Act, 2004). All of these efforts encourage greater use of the existing federallysponsored but voluntary repository of clinical trails, www.clinicaltrials.gov. We look forward to the eventual open access to all results regarding the efficacy of medication and other therapies as well as reports of adverse reactions experienced following regulatory approval.

\section{TREATMENT FOR SUBSTANCE ABUSE}

Treatment for drug dependence in adolescents raises a host of issues because some drugs of dependence (e.g., marijuana) are banned by law and their use is treated as criminal behavior. This is unfortunate because dependence on most drugs can be successfully treated if the family is involved in the therapy (see Chapter 18). Furthermore, drug dependence is often comorbid with other mental conditions that would benefit from treatment as a medical problem rather than as criminal behavior. Because the treatment system for substance abuse is not integrated with treatment for mental conditions (see below), those with both suffer needlessly.

\section{EARLY DETECTION AND TREATMENT AS A PREVENTION STRATEGY}

It is now clear that most cases of adult mental disorders make their first appearance prior to or during adolescence (Kim-Cohen, et al., 2003; Roza, Hofstra, van der Ende, \& Verhulst, 2003). This reality makes the early detection and treatment of mental disorders even more critical (see also The President's New Freedom Commission on Mental Health, 2003). The earlier a condition is identified and treatment begun, the less serious the course of illness and the lower the likelihood that it will disrupt healthy adolescent development. This is particularly important for substance dependence, including smoking, because there is evidence of nervous system plasticity during adolescence. A drug habit learned early produces brain changes that may be lifelong. If early detection and treatment of mental disorders were the norm, the possibility of reducing subsequent disorder would be increased. Furthermore, given the high rates of mental disorders as precursors to suicide, their early treatment would boost our chances of preventing this fatal outcome in youth.

Because it is clear that early detection and referral for treatment should be a high national priority, it is disappointing to learn from research conducted as part of the commissions (Chapters 30 and 31) that the primary care system and schools are inadequately prepared to meet this challenge. Primary care physicians are not trained to detect mental disorders or substance abuse problems, and most do not employ screening programs to identify youth at risk for these conditions. A similar situation exists in the schools where mental health professionals do not have the resources to identify youth at risk for problems. As a result, schools do not intervene until illnesses progress and come to the attention of staff. Unfortunately, the most common disorders in adolescence (depression, anxiety, and substance abuse) are not as easily recognized as conduct disorder and attentiondeficit hyperactivity disorder (ADHD), conditions that make their first appearance in the elementary years. Waiting until adolescent conditions seriously interfere with school performance forestalls treatment and reduces the odds of successful recovery.

Treatment systems also are poorly designed for delivering care to adolescents. The most glaring example of this, treatment for substance abuse, is a case study of inadequate response to a large but potentially manageable problem (Chapter 29). The long-standing dichotomy between treatment for drug dependence and other mental conditions creates a barrier that prevents comprehensive treatment. Since substance abuse 
and mental conditions often co-occur, they should be treated within the same service system. Furthermore, substance dependence treatment programs often employ ineffective interventions despite the existence of evidence-based therapies (involving the family) that can produce greater treatment adherence and success.

The integration of treatment services (mental health, addiction treatment, behavioral health) across different practitioners in different settings (primary care, specialty care, schools, juvenile justice) requires coordination that is difficult to achieve under the current health care system. New systems of treatment for mental health that integrate services are sorely needed. As noted in Chapter 28 by Hoagwood, several states, most notably Hawaii, Michigan, and New York, are attempting to implement new public programs that include evidence-based treatments and are coordinating structures to ensure that youth are effectively served. We await further evaluations of these programs to see if they can solve the service fragmentation problems that have plagued mental health treatment for both youth and adults.

\section{UNIVERSAL PREVENTION}

Aside from early detection of mental disorders, there is growing evidence that universal prevention programs in schools and other youth settings can help to increase resilience to stress and encourage healthy growth and decision making. The evidence in the case of drugs and alcohol as well as depression is particularly encouraging (see Chapters 3, 19, and 26). Suicide prevention programs are also showing encouraging signs (see Chapter 24). However, in our recommendations for further research (below), we observe that much remains to be learned about these programs.

\section{INSURANCE COVERAGE}

No discussion of treatment problems can ignore the weaknesses in insurance coverage for mental health. The Mental Health Parity law that went into effect in 1998 is due for re- newal in 2004, and several changes have been proposed in the latest legislation to make coverage of mental health conditions more inclusive. Achievement of full parity is an important policy goal. As a report by the Congressional Research Service notes (Redhead, 2003), "Requiring parity for mental health benefits establishes a uniform 'floor' of mental health coverage across all plans." This has the desirable effect of reducing wasteful competition among health-insurers to attract the least risky policyholders. In addition, experience in the implementation of full parity in federal health insurance programs indicates that the short-term increases in costs are small (about $1.6 \%$ in feefor-service plans and less than $1 \%$ in managed care) (Redhead, 2003). Hence, short-term cost does not appear to be a barrier to the implementation of full parity for mental health services, and the long-term cost savings and benefits to consumers should make parity costeffective.

Despite the importance of parity legislation, this policy effort also has serious limitations. At least 15\% of Americans have no health insurance coverage whatsoever (Mills \& Bhandari, 2003). This means that nearly $12 \%$ of youth under the age of 18 and $30 \%$ of young people between the ages of 18 and 24 without coverage will not be helped by parity. In addition, parity legislation affects only those whose insurance already includes coverage for mental health, and it does not cover treatment for substance dependence (Redhead, 2003). Hence, even if the current parity legislation were enacted, it would not solve the problems inherent in the current system of treatment for mental health.

A potentially favorable development in the search for solutions to inadequate mental health coverage is the prospect that managed care programs, which now cover more than half of youth (Glied \& Neufield, 2001), will evolve to adequately ensure treatment for mental conditions. One approach adopted by managed care involves the use of specialized services or "carveouts" for behavioral treatment. Through this approach, costs for mental health services can be contained while patients are directed to appropriate care by specialists (Conti, Frank, \& McGuire, 2004). In principle, the short-term costs of 
mental health services can be effectively managed. Nevertheless, it remains to be seen if managed care can adequately accommodate youth who require more long-term treatment to either complete a full course of psychotherapy or maintain treatment and avoid relapse (Glied \& Neufield, 2001; Kelleher, Scholle, Feldman, \& Nace, 1999).

Another challenge for managed care and other insurance plans is to identify those practitioners who can treat adolescent mental problems effectively. Surveys of both primary care providers and school mental health professionals indicate widespread dissatisfaction with the quality of care available in local communities for adolescents having the disorders studied by our commissions. The effectiveness of early detection and treatment of adolescent mental disorders is thus limited. There is an urgent need to increase the pool of providers able to deliver evidence-based treatments to youth so that availability of such services is no longer a barrier to referral by schools and primary care providers. School mental health professionals and nurses as well as primary care providers need links with quality treatment centers to which they can refer adolescents in need.

\section{STIGMA OF MENTAL DISORDER}

Research conducted by Penn and colleagues (Chapter 27) indicates that young people hold stigmatizing beliefs about mental disorders and that these beliefs can influence their treatment decisions. If youth feel that they will be stigmatized for seeking treatment, they will resist coming forward when experiencing symptoms. One approach to reducing this dysfunctional response is to increase public awareness of the efficacy of treatment for mental disorders. If adolescents (and their parents) recognize the potential effectiveness of early detection and treatment, they may be more inclined to seek help from providers who are seen as effective in treating their problems. Hence, through awareness of treatment efficacy, the effects of stigmatizing beliefs may be moderated and youth encouraged to seek help from providers.

Educating the public about the effectiveness of treatment and the reality that persons with mental disorders can lead productive lives is a strategy that deserves the support of the advocacy community and government agencies. The current mental health media campaign sponsored by the Substance Abuse and Mental Health Services Administration (SAMHSA) as part of its Elimination of Barriers Initiative (2004) is one example of such an effort.

\section{RESEARCH NEEDS}

Estimates of the economic costs of mental and substance abuse disorders in the United States are in excess of $\$ 200$ billion per year (Redhead, 2003; U.S. Department of Health and Human Services, 1999). By increasing our ability to prevent and treat these disorders in young people, we have the potential to reduce this burden significantly while substantially increasing the nation's welfare. We see two kinds of research opportunities for improving our ability to prevent and treat mental disorders in youth. At the procedural level, there are many unanswered questions about appropriate protocols and systems for treatment. Although we have treatments that work, we still don't know enough about the best ways to combine treatments for maximum effect or the optimal ways to withdraw treatments without leading to relapse. We also need more research on the best ways to treat persons with comorbid conditions, especially addiction. Moreover, we have no direct evidence of efficacy for the treatment of some adolescent anxiety and eating disorders, and schizophrenia still remains a treatment challenge.

We also know that universal prevention of some conditions is possible (Chapters 3 and 19) and that the healthy development of youth can be enhanced considerably using positive youth development strategies (see Chapter 26). Nevertheless, much remains to be learned about integrating effective programs into schools and communities in a cost-effective way. The promise of positive psychology is still untested and requires more research.

We also lack knowledge of the best ways to conduct screening of youth in schools and primary care settings so that care is delivered to 
them. Many young people identified in screening programs as needing services fail to seek them. There are several promising models (discussed by the suicide commission) but not enough evidence about their efficacy or ability to be taken to scale to permit widespread adoption.

A second major opportunity for research is to increase our understanding of the multiple pathways to both healthy and unhealthy development. Because we know little about the ways in which disorders unfold, it is difficult to design appropriate interventions at the earliest signs of disorder. One shortcoming in previous research is that investigators inevitably focused on the one or two disorders in which they had expertise. Unfortunately, the many disorders that appear in adolescence do not accommodate this neat developmental pattern. Disorders that first appear in childhood may later develop into other forms and some may develop together (Kim-Cohen et al., 2003). Early emergence of some conditions may be left behind never to appear again. These complex patterns of comorbidity and development have been a frequent finding in the commission reports. The challenge for future research is to examine a broad range of potential psychopathology as well as sources of resilience in one design in which different developmental pathways can be studied at the same time.

Fortunately, research strategies to better understand the causal influences on varied developmental trajectories are available (Curtis \& Cicchetti, 2003; Masten, 2004). Longitudinal studies of representative populations can help to identify the paths and influences that affect the emergence of a disorder (e.g., Caspi et al., 2002; Kim-Cohen et al., 2003). Studies of twins embedded in such studies make it possible to identify genetic and interacting environmental factors that alter trajectories toward either resilience or disability (Rutter et al., 1997; Waldman, 2003). Once potential causal influences have been isolated, their effects can be studied in more focused longitudinal designs that select high-risk youth for prospective investigation. Clinical trials that follow young people over time can test the effects of theoretically derived interventions, thereby adding further knowledge of causally efficacious treatments (Masten, 2004).

A research project designed to follow a large representative sample of children and twins that will enable the study of different developmental pathways throughout the early years and into adulthood is prohibitively expensive, even for the National Institute of Mental Health. Funding for such an endeavor will require the collaboration of a multitude of investigators with different areas of expertise, and it will necessitate the cooperation of both federal and private funding sources. It is clear, however, that such a study would greatly advance our understanding of the emergence of mental disorder and resilience in youth and allow further tests of intervention strategies that could reduce the burden of mental disorders in adolescence and adulthood.

\section{THE FUTURE}

With recent advances in neuroscience, it is now clear that adolescence is a period of dramatic change in brain structure and function (Giedd, 2004; Spear, 2000). The brain is an eminently plastic organ that develops both in accord with genetic rules and in response to its environment (Huttenlocher, 2002). Adolescence is particularly critical because it is the period during which significant "pruning" of synaptic connections occurs. The connections that remain may allow experience to mold a more adaptive brain, but they also open the door to illness and dysfunctional behavior. We remain far from understanding the mechanisms that produce these varied outcomes, but the potential for therapeutic interventions to correct dysfunctional neurodevelopment and to enhance resilience is a clear possibility. As we learn more about these effects, possibilities for dramatic breakthroughs in our ability to prevent and alter dysfunctional trajectories will present themselves (Kandel, 1998).

Our new understanding of brain development is rendering the old debates between nature and nurture or biology vs. behavior increasingly irrelevant (Kandel, 1998). Gene expression is responsive to environments and hormonal changes. As Kandel (1998) put it, "nurture will 
become nature," and our ability to influence such developments in positive ways will greatly enlarge the scope of preventive interventions. Increasing evidence of brain plasticity during the adolescent years suggests that both biologically and socially based interventions can influence development and alter dysfunctional developmental paths. The battleground between oppos- ing biological and environmental viewpoints may be merging into a synthesis that recognizes the value and promise of both sides of these debates. These new frontiers in the study of psychopathology make this a most exciting time for advancing our knowledge and ultimately for preventing the onset and development of mental disorders at the earliest signs of presentation. 
This page intentionally left blank 